\title{
Blast Cell Methotrexate-Polyglutamate Accumulation In Vivo Differs by Lineage, Ploidy, and Methotrexate Dose in Acute Lymphoblastic Leukemia
}

\author{
Timothy W. Synold, Mary V. Relling, James M. Boyett, Gaston K. Rivera, John T. Sandlund, Hazem Mahmoud, \\ William M. Crist, Ching-Hon Pui, and William E. Evans \\ Hematology-Oncology, Pharmaceutical, and Biostatistics Departments, St. Jude Children's Research Hospital; \\ and Departments of Pediatrics and Clinical Pharmacy, University of Tennessee, Memphis, Tennessee 38101
}

\begin{abstract}
High-dose methotrexate (HDMTX) is a component of most treatment protocols for childhood acute lymphoblastic leukemia (ALL), yet recent studies of receptor-mediated transport and saturable polyglutamylation have questioned its rationale. To investigate this in vivo, methotrexate and its active polyglutamated metabolites (MTX-PG) were measured in bone marrow blasts obtained from 101 children randomized to single-agent therapy with either HDMTX (1 g/m $\mathrm{m}^{2}$ per $24 \mathrm{~h}$ i.v., $n=47$ ) or low-dose MTX (LDMTX, 30 $\mathrm{mg} / \mathrm{m}^{2}$ by mouth every $6 \mathrm{~h} \times 6, n=54$ ), before remission induction therapy. Blast concentrations of total MTX-PGs (median 460 vs 1380 pmol/10 cells) and of long-chain MTXglu $_{4-6}$ were both significantly higher after HDMTX $(P<$ 0.001). With either treatment, MTX-PGs were significantly higher in B-lineage blasts than in T-lineage blasts (LDMTX $P=0.001$, HDMTX $P=0.03$ ). In a multiple regression analysis of B-lineage ALL, blast MTX-PG was significantly related to MTX dose (or plasma MTX concentration), lymphoblast ploidy (hyperdiploid > nonhyperdiploid), and percentage S-phase. This is the first evidence that HDMTX achieves higher MTX-PG concentrations in ALL blasts in vivo, establishing a rationale for HDMTX in the treatment of childhood ALL, especially T-lineage or nonhyperdiploid B-lineage ALL, disease characteristics associated with a poor prognosis on conventional therapy. (J. Clin. Invest. 1994. 94:1996-2001.) Key words: T-lineage leukemia $\cdot$ hyperdiploidy $\bullet$ folylpolyglutamate synthetase
\end{abstract}

\section{Introduction}

Despite several decades of clinical evaluation, the optimal dosage of methotrexate (MTX) ${ }^{1}$ for the treatment of childhood acute lymphoblastic leukemia (ALL) remains controversial. While most investigators agree that weekly administration of

Address correspondence to Dr. William E. Evans, St. Jude Children's Research Hospital, Memphis, TN 38105. 1994.

Received for publication 13 May 1994 and in revised form 11 July

1. Abbreviations used in this paper: $\mathrm{ALL}$, acute lymphoblastic leukemia; DHFR, dihyrofolate reductase; DI, DNA index; FPGS, folylpolyglutamate synthetase; HDMTX, high-dose methotrexate; LDMTX, low-dose methotrexate; MTX, methotrexate; MTX-PG, methotrexate polyglutamates; MTX-glu ${ }_{n}$, methotrexate with $n$-glutamyl residues.

J. Clin. Invest.

(C) The American Society for Clinical Investigation, Inc. 0021-9738/94/11/1996/06 \$2.00

Volume 94, November 1994, 1996-2001 low doses (e.g., $40 \mathrm{mg} / \mathrm{m}^{2}$ ) is appropriate for therapy during remission, there is no consensus regarding the rationale for highdose MTX (e.g., $1000 \mathrm{mg} / \mathrm{m}^{2}$ i.v. over $24 \mathrm{~h}$ ) as a component of ALL treatment. Although several treatment regimens with improved outcome included high-dose MTX (HDMTX) (1-3), in vitro studies have suggested that low-dose MTX (LDMTX) can achieve comparable intracellular concentrations at lower cost and with less inconvenience (4-6). Investigators favoring LDMTX argue that receptor-mediated MTX transport via potocytosis (7) is maximal at extracellular MTX concentrations of $0.05-0.1 \mu \mathrm{M}$ in receptor-positive cells, and that chronic exposure to low concentrations will achieve intracellular concentrations comparable to HDMTX treatment (6). Those advocating HDMTX contend that higher extracellular MTX concentrations will lead to increased entry of MTX into cells by alternative mechanisms (e.g., passive diffusion), including resistant blasts with reduced active membrane transport of MTX. Consistent with the latter view, we previously reported a lower risk of treatment failure in children with ALL in whom plasma MTX concentrations were above $16 \mu \mathrm{M}$ following HDMTX therapy, indicating the potential importance of achieving higher extracellular MTX concentrations $(1,8)$.

Advocates of LDMTX also argue that high concentrations of MTX may impair formation of MTX-polyglutamates (MTXPG) through feedback inhibition, as has been demonstrated in vitro (9-11). Metabolism of MTX to its polyglutamated metabolites, especially long-chain polyglutamates (i.e., MTX-glu $\mathbf{M}_{4-6}$ ), is considered important for maximum pharmacologic effects (12). These active MTX-PG metabolites are formed by folylpolyglutamate synthetase (FPGS), the enzyme that catalyzes synthesis of polyglutamated forms of natural folates as well as antifols such as MTX. Methotrexate polyglutamates, particularly those with more than three glutamyl residues, are retained in cells longer than MTX, providing a mechanism by which MTX-PGs may produce greater cytotoxicity (13-16). Moreover, MTX-PGs inhibit not only dihydrofolate reductase (DHFR) $(17,18)$, they also inhibit other folate-dependent enzymes that are not substantially inhibited by MTX, such as thymidylate synthase (19) and transformylases required for de novo purine synthesis (20). The absence of MTX-PG formation has been established as a mechanism by which cancer cells can develop resistance to MTX (21-24). In this regard, Whitehead et al. (25) demonstrated that children with B-lineage ALL have a more favorable prognosis if their leukemic blasts accumulate higher concentrations of MTX-PG after in vitro incubation with MTX.

This study is the first to establish in vivo that HDMTX achieves higher blast MTX-PG concentrations than LDMTX, and that MTX-PG accumulation is significantly lower in Tlineage and nonhyperdiploid ALL blasts, both features known to carry a worse prognosis. Moreover, HDMTX produced blast MTX-PG concentrations in T-lineage and nonhyperdiploid pa- 


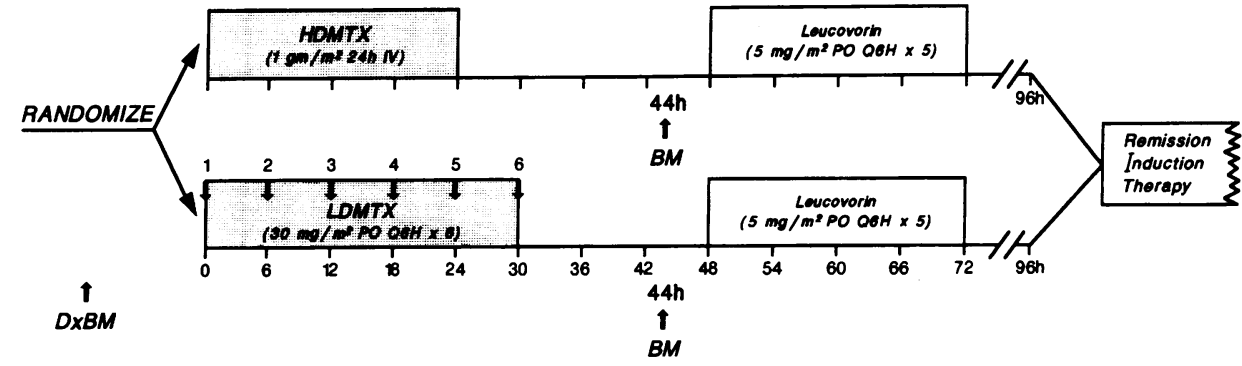

Figure 1. Schema of MTX and leucovorin administration and timing of bone marrow aspirates to obtain leukemic blasts for measurement of MTX-PG. BM, bone marrow aspirate at diagnosis (Dx) and $44 \mathrm{~h}$; HDMTX infused intravenously over $24 \mathrm{~h}$. LDMTX given orally at times indicated by downward arrows. tients comparable to those achieved in B-lineage or hyperdiploid blasts with LDMTX, suggesting HDMTX may circumvent the inherent problems with reduced MTX-PG accumulation, and possibly improve outcome in this subgroup of ALL patients known to have a poor prognosis with conventional therapy (26-28).

\section{Methods}

Patients. All patients aged $18 \mathrm{yr}$ and younger with newly diagnosed acute lymphoblastic leukemia, excluding those with a mature B cell phenotype, were enrolled on the study between December 1991 and September 1993. The diagnosis of acute lymphoblastic leukemia was based on morphology, cytochemical staining properties, and immunophenotyping of blast cells (27). The blasts were further subclassified as T-lineage (cytoplasmic $\mathrm{CD}^{+}, \mathrm{CD}^{+}$, plus $\mathrm{CD}^{+}$, or $\mathrm{CD}^{+}$, or both) or B-lineage (cytoplasmic $\mathrm{CD} 22^{+}, \mathrm{CD} 19^{+}$, $\mathrm{HLA}-\mathrm{DR}^{+}, \mathrm{CD} 10^{ \pm}$), as previously described (27). A flow cytometric analysis was performed to determine the percent of leukemic blast cells in S-phase and to determine ploidy based on the DNA index (DI; ratio of DNA content in leukemic G0/G1 cells versus normal diploid G0/G1 cells) (28). Bone marrow samples for chromosome analysis were prepared by the method of Williams and colleagues (29); metaphases were G-banded by treatment with trypsin and studied with Wright's stain. Chromosomal abnormalities were classified according to the International System for Human Cytogenetic Nomenclature (30). Signed informed consent was obtained from parents and(or) patients.

Study protocol. All studies were conducted during the initial $96 \mathrm{~h}$ after diagnosis, before initiation of conventional remission induction therapy (Fig. 1). Once enrolled, patients were randomized to receive either high-dose methotrexate (HDMTX) or fractionated low-dose methotrexate (LDMTX) as the only antileukemic agent during this interval. HDMTX consisted of a $200-\mathrm{mg} / \mathrm{m}^{2}$ i.v. push over $5 \mathrm{~min}$, followed by $800 \mathrm{mg} / \mathrm{m}^{2}$ i.v. over $24 \mathrm{~h}$, while the LDMTX consisted of $30 \mathrm{mg} / \mathrm{m}^{2}$ by mouth every $6 \mathrm{~h}$ for six doses. HDMTX patients were prehydrated $\geq 2$ $\mathrm{h}$ before the MTX infusion with $5 \%$ dextrose/ 0.25 normal saline and 40 meq $\mathrm{NaHCO}_{3} /$ liter, until the urine specific gravity was $<1.015$ and urine $\mathrm{pH}$ was $\geq 6.5$. Both treatment groups were given identical leucovorin rescue, $5 \mathrm{mg} / \mathrm{m}^{2}$ by mouth every $6 \mathrm{~h} \times 5$ doses, beginning $48 \mathrm{~h}$ after the start of MTX. If the 44-h MTX plasma concentration was $>1.0 \mu \mathrm{M}$, the dosage of leucovorin was increased and continued until plasma MTX was $<0.1 \mu \mathrm{M}$.

On day 4, all patients began conventional remission induction therapy with six drugs (i.e., prednisone, vincristine, daunomycin, asparaginase, etoposide, and cytosine arabinoside) given over $29 \mathrm{~d}$ in the dosages and schedules previously described in detail (31). The use of etoposide $\left(300 \mathrm{mg} / \mathrm{m}^{2}\right.$ per dose) instead of teniposide, and the omission of daunomycin on day 15 , were the only major changes from the previously reported remission induction therapy (31).

Blood sample collection and analysis. Blood was obtained before and at $1,6,23,44$, and $68 \mathrm{~h}$ after the start of HDMTX infusion. In patients receiving LDMTX, blood was obtained before doses 1 and 2; before and 1, 1.5, 2, and $6 \mathrm{~h}$ after dose 4; and then at 14 and $38 \mathrm{~h}$ after dose 6. Plasma samples were analyzed for MTX by a fluorescence polarization immunoassay (Abbott $\mathrm{TD}_{\mathbf{x}}$; Abbott Laboratories, North
Chicago, IL). Pharmacokinetic parameters were estimated assuming a first-order two compartment model, using a Bayesian estimation algorithm, as implemented in ADAPT II software (32). The prior distribution for model parameters was based on previous data in children who received HDMTX or $\operatorname{LDMTX}(1,33)$. To assess the relationship between MTX systemic exposure and leukemic blast MTX-polyglutamate concentrations, the MTX steady-state plasma concentration at hour 23 of the 24-h infusion was used as the measure of systemic exposure in patients receiving HDMTX, while the model estimated steady-state peak MTX concentration (after dose 4) was used in the LDMTX group.

Bone marrow sample collection and analysis. To measure MTXPG concentrations in ALL blasts, a bone marrow aspirate was performed $44 \mathrm{~h}$ after the start of MTX therapy, before leucovorin rescue. Samples of $2-10 \mathrm{ml}$ of bone marrow were collected in syringes containing 800 $\mathrm{u}$ of heparin. Leukemic blast cells were isolated by Ficoll-Hypaque gradient and washed three times in cold culture media (RPMI 1640 containing $10 \%$ fetal bovine serum and $2 \mathrm{mM}$ L-glutamine). The final cell yield was determined by hemacytometer and percent viability by trypan blue exclusion. Cells $\left(\geq 5 \times 10^{6}\right)$ were extracted for analysis using a modification of the method of Kamen and Winick (34).

Methotrexate and six polyglutamated metabolites (MTX-glu ${ }_{2}$ to MTX-glu ${ }_{7}$ ) were separated using a previously reported HPLC method (35). The column eluent was collected in fractions determined by the elution times of each polyglutamated metabolite (MTX-glu ${ }_{2}$ to MTX$\mathrm{glu}_{7}$, obtained from Schircks Laboratories, Jona, Switzerland). Each fraction was dried to completion and assayed using a radio-ligand binding assay (The Enzyme Center, Inc., Malden, MA) (36). Separate calibration curves were used for quantitation of MTX and each polyglutamated metabolite. The limit of detection of this assay was $0.02 \mathrm{pmol} /$ $10^{6}$ cells; all results were expressed as picomoles of MTX or MTX-PG per $10^{9}$ cells (for comparison to published in vitro data normalized to $10^{9}$ cells).

Statistics. The statistical design for the primary question (HDMTX vs LDMTX) called for 70 children to be stratified by age at diagnosis ( $\leq 5 \mathrm{vs}>5 \mathrm{yr}$ ), DI $(<1.16$ or $>1.6$ vs $1.16-1.60)$, and white blood cell count $(<25,000$ vs $\geq 25,000$ cells $/ \mu \mathrm{l})$ and randomized to receive either HDMTX or LDMTX. This design had $90 \%$ power $(\alpha=0.01$, two sided test) to detect a $50 \%$ difference in mean concentrations of total MTX-PGs. The method of Fleming, et al. (37) was used to plan one interim analysis at a significance level of 0.003 after 35 patients had been randomized and the final analysis at a level of 0.00791 . The data were analyzed using a stratified Wilcoxon statistic. The interim analysis revealed a significant difference between HDMTX and LDMTX $(P=0.0018$, exact test), however, additional patients were enrolled to assess patient and disease characteristics associated with the observed variability in MTX-PG within each treatment group. Presenting features were compared between the two treatment groups using the Chi-squared statistic or Fisher's exact test, as appropriate.

\section{Results}

Patients. From December 1991 to September 1993, 109 patients were randomized; 101 (93\%) of whom completed the study and were evaluable. Of the eight patients not evaluable, four were considered too unstable to complete the studies, two refused 
Table I. Demographics of Study Population

\begin{tabular}{lccc}
\hline & $\begin{array}{c}\text { LDMTX } \\
(n=54)\end{array}$ & $\begin{array}{c}\text { HDMTX } \\
(n=47)\end{array}$ & $P$ value* \\
\hline Age $($ yr $)$ & & & \\
$<1$ & 0 & 1 & \\
$1-10$ & 38 & 36 & 0.37 \\
$>10$ & 16 & 10 & \\
WBC $\left(\times 10^{9}\right.$ /liter $)$ & & & \\
$<50$ & 38 & 35 & \\
$50-100$ & 7 & 3 & 0.55 \\
$\geq 100$ & 9 & 9 & \\
Sex & & & \\
Male & 29 & 27 & \\
Female & 25 & 20 & \\
DNA index & & & \\
$1.16-1.6$ & 12 & 7 & \\
$<1.16$ or $>1.6$ & 42 & 40 & \\
Ploidy & & & \\
$>50$ chromosomes & 13 & 9 & \\
$\quad \leq 50$ chromosomes & 40 & 36 & \\
Not determined & 1 & 2 & \\
Immunophenotype & & & \\
B-lineage & 44 & 43 & \\
T-lineage & 10 & 4 & \\
& & & \\
\hline
\end{tabular}

* Fisher's exact test.

the additional bone marrow aspirate, one was diagnosed as having acute myelogenous leukemia after being enrolled, and one patient had a significant interruption of the MTX infusion. There were no significant differences in distributions of age, sex, WBC, lymphoblast ploidy, and blast cell immunophenotype between the two treatment groups (Table I). There was also no significant difference between the two treatment groups in percentage of blasts in the diagnostic marrow sample (median, $\mathrm{LD}=90 \%, \mathrm{HD}=91 \%$ ) or in the 44 -h bone marrow sample (median, $\mathrm{LD}=90 \%$, HD $=89 \%$ ). Likewise, the percentage of viable cells in the 44-h marrow samples (post-Ficoll-Hypaque separation) did not differ between the two groups (median; LD $=98 \%$; HD $=97 \%$ ). On day four after MTX therapy, 4 of 41 (9.8\%) LDMTX patients versus 7 of 34 (20.6\%) HDMTX patients had complete clearing of circulating blasts.

Methotrexate plasma pharmacokinetics. MTX elimination half-lives were similar in both treatment groups [median (range); LDMTX, $6.4 \mathrm{~h}$ (5.4-27); HDMTX, $6.6 \mathrm{~h}$ (5.3-22)]. There was approximately a 12-fold difference in the maximum steady state plasma concentrations [median (range); LDMTX, $0.9 \mu \mathrm{M}$ [0.2-20]; HDMTX, $12.2 \mu \mathrm{M}$ (5.4-41); $P<0.001$ ], while the median time plasma MTX remained above $0.1 \mu \mathrm{M}$ before the bone marrow aspirate was $42.8 \mathrm{~h}$ for LDMTX (range 30-44 h) and $44 \mathrm{~h}$ for HDMTX (range: $40-44 \mathrm{~h}$ ). Fig. 2 depicts plasma concentration-time data and model simulations using pharmacokinetic parameters from typical patients in the two treatment groups.

Intracellular methotrexate polyglutamates. The median blast MTX and MTX-PG concentrations for the 101 evaluable patients are summarized in Fig. 3. Total blast MTX-PG concentrations were significantly higher in patients treated with HDMTX versus LDMTX (1380 vs. $460 \mathrm{pmol} / 10^{9}$ cells; $P$ $=0.001) .83 \%$ of HDMTX patients but only $44 \%$ of LDMTX

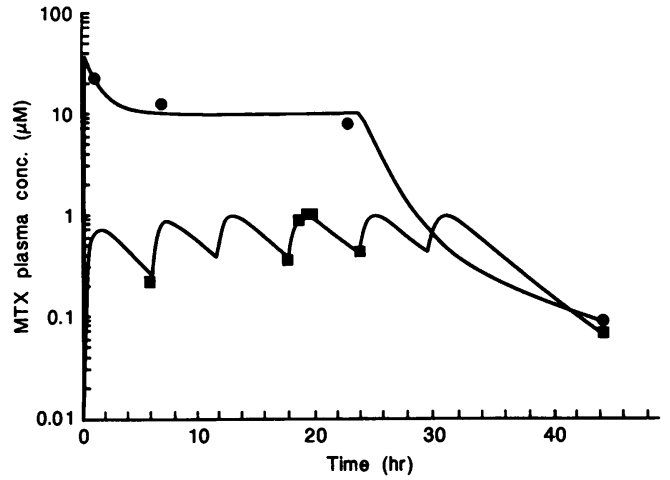

Figure 2. Plasma concentration versus time for MTX in a typical patient treated with high dose intravenous MTX (.) and low dose oral MTX (घ). Lines depict model simulations using pharmacokinetic parameters for each patient. Symbols represent actual measured concentrations.

patients $(P=0.0002)$ had total MTX-PG $>500 \mathrm{pmol} / 10^{9}$ cells, an in vitro level previously associated with a favorable outcome in ALL (25). Patients treated with HDMTX also had significantly higher concentrations of long-chain MTX-PGs (i.e., MTX-glu $_{4-6}, 986$ vs $355 \mathrm{pmol} / 10^{9}$ cells; $\left.P=0.001\right)$. MTX-glu was the predominant MTX-PG in both HDMTX (60\%) and LDMTX $(65 \%)$ patients, while MTX-glu 6 was the predominant MTX-PG in $15 \%$ of HDMTX patients versus $3.7 \%$ of LDMTX patients. MTX-glu ${ }_{7}$ was detectable in most patients $(81 \%)$, however, it represented $<2 \%$ of the total MTX-PG in both treatment groups and levels were typically near the limit of assay quantitation.

While the median MTX-PG concentration was threefold higher in patients treated with HDMTX compared to LDMTX, there was substantial interindividual variability within both treatment groups. As depicted in Fig. 3 (circles), a small number of patients treated with LDMTX $(n=5,9 \%)$ had blast MTXPG concentrations comparable to the median for the HDMTX group, and a small number of HDMTX patients $(n=6,13 \%)$ had blast MTX-PG below the median for LDMTX patients.

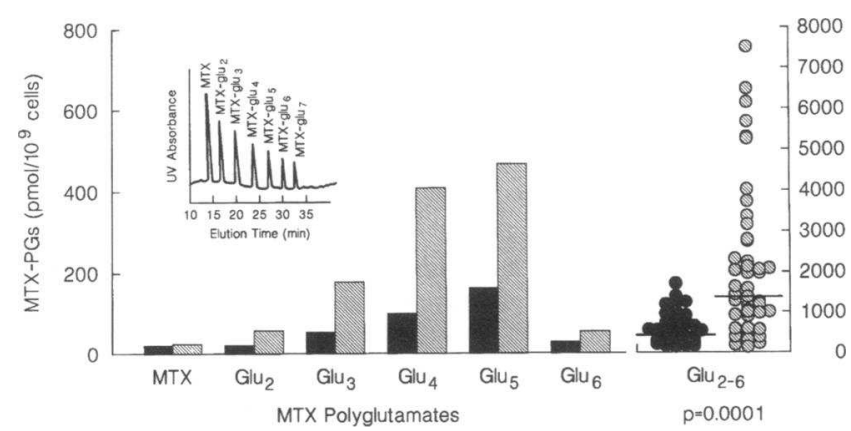

Figure 3. Median in vivo concentrations of MTX and MTX-PGs in leukemic blasts obtained $44 \mathrm{~h}$ after either high dose MTX (shaded bars) or low dose MTX (solid bars). Glu ${ }_{n}$, number of glutamate residues; MTX, methotrexate $\left(\mathrm{glu}_{1}\right)$. MTX-glu ${ }_{5}$ was the predominant MTX-PG

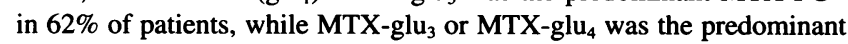
form in $11.8 \%$ and $12.7 \%$ of patients, respectively. Circles depict total MTX-PG (i.e., MTX-glu ${ }_{2-6}$ ) measured in blasts from each patient, horizontal bars depict median value in each group, $P$ value indicates statistical significance in total MTX-PG in HDMTX versus LDMTX. (Inset) HPLC separation of MTX and each polyglutamate metabolite. 
Table II. Univariate Analysis of Variables Potentially Associated with Blast MTX-PG Concentrations

\begin{tabular}{lc}
\hline \multicolumn{1}{c}{ Variable } & $P$ value \\
\hline MTX dose (LD vs. HD) & $<0.0001$ \\
MTX C $\mathrm{p}_{\max }(\mu \mathrm{M})$ & $<0.0001$ \\
Lineage $(\mathrm{B}$ or T) & $<0.0001$ \\
Ploidy & 0.006 \\
Percent S-phase & 0.007 \\
Age at diagnosis & NS \\
WBC at diagnosis & NS \\
Sex & NS \\
\hline
\end{tabular}

MTX, methotrexate; $\mathrm{LD}$, low dose; $\mathrm{HD}$, high dose; $\mathrm{Cp}_{\max }$, maximum plasma concentration; WBC, white blood cell count.

Characteristics related to MTX-polyglutamate concentrations in ALL blasts. The basis of interpatient variability in blast MTX-PGs was explored by analysis of patient and blast cell characteristics. When assessed by univariate analysis, higherdose, higher MTX plasma concentration, B-lineage, hyperdiploidy ( $>50$ chromosomes) and higher \%S were each significantly associated with higher blast MTX-PG concentrations (Table II).

Given the relatively small number of hyperdiploid T-lineage patients, multiple regression analysis was limited to B-lineage patients $(n=87)$. Since MTX dose and plasma concentration were correlated, they were not included simultaneously in the model; however, the final model was the same whether MTX dose (HDMTX vs LDMTX) or plasma concentration $(<5 \mu \mathrm{M}$ vs $\geq 5 \mu \mathrm{M}$ ) was included. In either case, higher dose (or plasma concentration), hyperdiploidy, and higher \%S were significantly associated with higher blast MTX-PG concentration $\left(r^{2}=0.42\right.$, $P<0.0001 ; n=76,11$ patients did not have $\%$ S determined).

Differences in MTX-PG concentrations in B-lineage versus $T$-lineage lymphoblasts. Since hyperdiploid blasts accumulated higher MTX-PG concentrations than non-hyperdiploid blasts (Table II) and hyperdiploidy is rare in T-lineage ALL, further assessment of lineage differences was confined to nonhyperdiploid ALL ( $n=76 ; 63$ B-lineage and 13 T-lineage).

As depicted in Fig. 4, the median blast MTX-PG concentration was significantly higher in B-lineage blasts than T-lineage blasts within each dosage group (LDMTX, 474 vs. $149 \mathrm{pmol} /$ $10^{9}$ cells, $P=0.001$ and HDMTX, 1413 vs. $552 \mathrm{pmol} / 10^{9}$ cells, $P=0.03$ ). Long-chain MTX-glu M $_{4-6}$ concentrations were significantly higher in B-lineage than in T-lineage blasts following LDMTX therapy (median 351 vs $51, P<0.001$, Fig. 4), while the lineage difference was not significant after HDMTX (median 1025 vs $437, P=0.13$ ). Patients with T-lineage ALL treated with HDMTX had blast MTX-PG concentrations similar to those achieved in B-lineage patients treated with LDMTX (577 vs. $488 \mathrm{pmol} / 10^{9}$ cells, $P=0.78$ ). This was also true for long-chain MTX-glu M-6 $_{4}\left(442\right.$ vs. $323 \mathrm{pmol} / 10^{9}$ cells, $\left.P=0.67\right)$.

Leukemic ploidy and blast MTX-PG concentrations. Assessment of leukemic cell ploidy as a determinant of MTX-PG accumulation was limited to only B-lineage ALL, due to the low prevalence of hyperdiploidy ( $>50$ chromosomes) in Tlineage ALL and the significant difference between T-lineage and B-lineage blasts in the accumulation of MTX-PG (as described above). As shown in Fig. 4, blasts from patients with hyperdiploid ALL $(n=21)$ accumulated higher concentrations of MTX-PGs than blasts from patients with nonhyperdiploid ALL $(n=63)$ (LDMTX: 758 vs. 474 pmol $/ 10^{9}$ cells, $P=0.01$; HDMTX: 3371 vs. $1413 \mathrm{pmol} / 10^{9}$ cells, $\left.P=0.055\right)$. Although MTX-glu ${ }_{5}$ was the predominant polyglutamate in hyperdiploid and nonhyperdiploid patients treated with either HDMTX or LDMTX, hyperdiploid blasts accumulated significantly higher concentrations of long-chain $\mathrm{MTX}-\mathrm{glu}_{4-6}$ in both treatment groups $(P<0.04$ and $P<0.01$, respectively). Nonhyperdiploid ALL patients treated with HDMTX had blast MTX-PG concentrations similar to those achieved in hyperdiploid blasts treated with LDMTX (1339 vs. $832 \mathrm{pmol} / 10^{9}$ cells; $P=0.09$ ); this was also true for long-chain MTX-glu M-6 $(995 \mathrm{vs} .736 \mathrm{pmol} /$ $10^{9}$ cells; $P=0.30$ ). There was no single chromosomal duplication that correlated with higher blast MTX-PG among hyperdiploid cases. The mean (range) fraction of cells in S-phase was significantly higher in hyperdiploid blasts $8.9 \%$ (1.5-16.3), when compared to nonhyperdiploid blasts $4.8 \%$ (0.4-27.6); however, both ploidy and $\% \mathrm{~S}$ were significant independent variables in the multiple regression model, indicating that higher MTX-PG in hyperdiploid blasts was not entirely due to differences in $\% \mathrm{~S}$.

\section{Discussion}

This study has established that HDMTX $\left(1 \mathrm{~g} / \mathrm{m}^{2}\right.$ infused i.v. over $24 \mathrm{~h}$ ) produces higher concentrations of MTX-polyglutamates in leukemic blast cells in vivo, when compared to those achieved by fractionated oral LDMTX. Although both regimens maintained plasma MTX concentrations above $0.1 \mu \mathrm{M}$ for a similar duration of time (median 42.8 versus $44 \mathrm{~h}$ ), the HDMTX regimen achieved MTX plasma concentrations that were $\sim 12-$ fold greater than the LDMTX regimen. This indicates that it was the higher plasma concentrations, and not a longer duration of exposure $>0.1 \mu \mathrm{M}$, that led to the difference in intracellular MTX-PG concentrations. Importantly, HDMTX produced significantly higher concentrations of long-chain MTX-polyglutamates (i.e., 4-6 glutamyl residues), when compared to LDMTX. In vitro studies $(10,11,38)$ have shown qualitative changes in the nature of polyglutamates, with a shift toward shorter chain polyglutamates, i.e., $\mathrm{Glu}_{2}$, when FPGS substrate concentrations are high, but this was not observed in vivo over the concentration range evaluated in the present study.

In light of recent studies demonstrating a more favorable clinical outcome in ALL patients whose blasts accumulated $>500 \mathrm{pmol} / 10^{9}$ cells after in vitro incubation with MTX, the ability to accumulate higher blast MTX-PG concentrations in vivo may represent an important therapeutic advantage for HDMTX. It is noteworthy that total MTX-PG exceeded 500 pmol $/ 10^{9}$ cells in $83 \%$ of HDMTX patients but in only $44 \%$ of LDMTX patients $(P=0.0002)$. Moreover, in T-lineage patients, 3 of 4 treated with HDMTX but none of 10 treated with LDMTX had MTX-PG $>500$ pmol $/ 10^{9}$ cells $(P<0.01$, Fig. 5), while in B-lineage patients, $84 \%$ with HDMTX versus $57 \%$ with LDMTX had MTX-PG $>500$ pmol $/ 10^{9}$ blasts $(P<0.001$, Fig. 5).

Patients with T-lineage ALL and those with nonhyperdiploid ALL generally fare worse than other patients treated with antimetabolite-based chemotherapy regimens (39). In the present study, T-lineage blasts accumulated lower MTX-PG than B-lineage blasts, and nonhyperdiploid B-lineage blasts accumulated significantly lower MTX-PG than hyperdiploid Blineage blasts, with either MTX dosage. Our in vivo findings are consistent with the in vitro data of Whitehead et al. (40), 

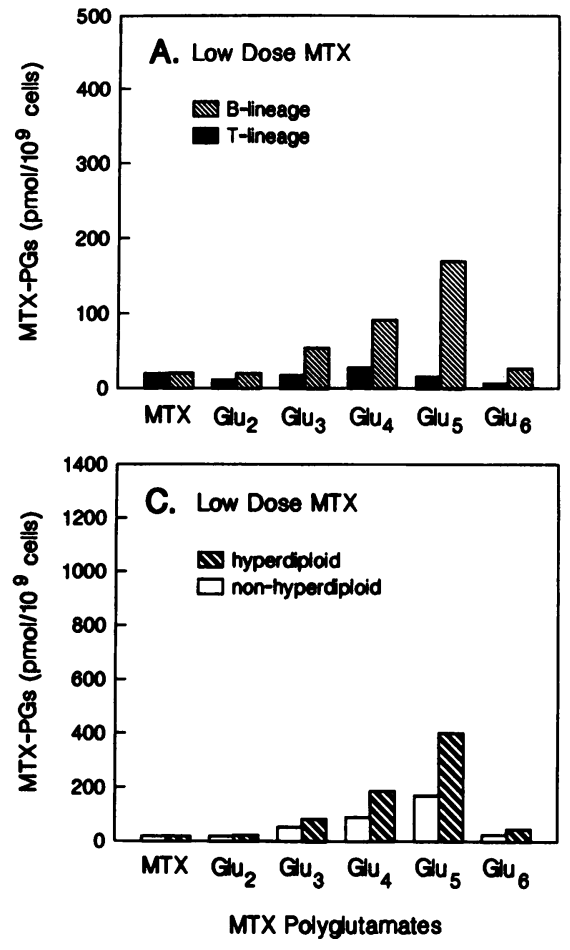
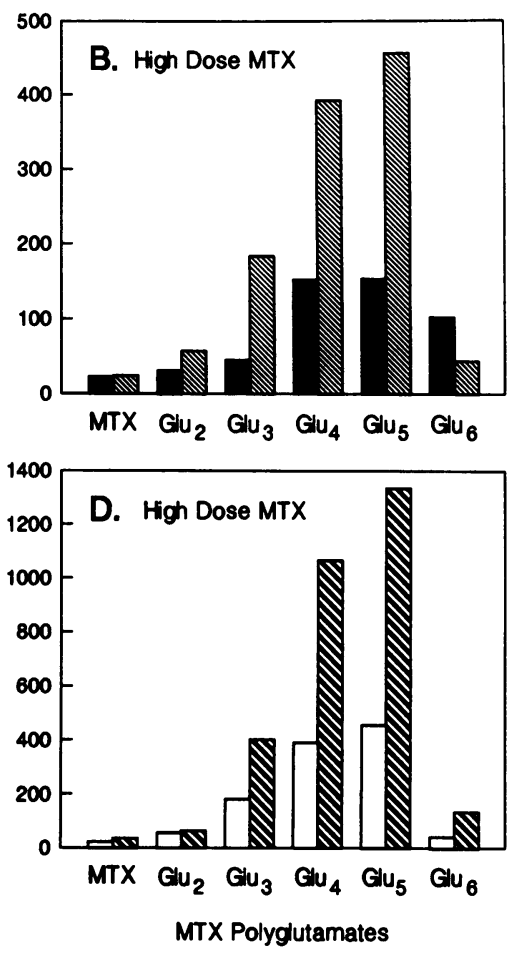

Figure 4. $A$ and $B$ depict median MTX-PGs in leukemic blasts from patients with B-lineage ALL (shaded bars, $n=76$ ) and T-lineage ALL (solid bars, $n=13$ ), after treatment with either LDMTX $(A)$ or HDMTX $(B)$. Hyperdiploid patients were excluded from the lineage comparison. $C$ and $D$ depict median MTX-PGs in leukemic blasts from B-lineage ALL patients with hyperdiploid blasts ( $>50$ chromosomes, hatched bars, $n$ $=21$ ) and nonhyperdiploid blasts (i.e., $\leq 50$ chromosomes, open bars, $n=63$ ), after either LDMTX $(C)$ or HDMTX $(D)$. who reported higher MTX-PG accumulation in hyperdiploid Blineage blasts incubated with MTX, and with the in vitro data of Goker et al. (41), who reported decreased formation of MTXPG in T-lineage blasts. Differences in MTX transport (cellular uptake), FPGS activity and/or degradation of MTX-PG by gamma-glutamyl hydrolase could explain the higher accumulation of MTX-PG in B-lineage blasts. In this regard, we have recently found that FPGS activity consistently increases in ALL blasts during MTX therapy, but that the median increase is only $37 \%$ in $\mathrm{T}$-lineage blasts compared to $188 \%$ in B-lineage blasts (42). Among patients with B-lineage ALL, our preliminary studies have not revealed a significant difference in FPGS activity in hyperdiploid (median FPGS $=905 \mathrm{pmol} / \mathrm{h}$ per $\mathrm{mg}$ protein,

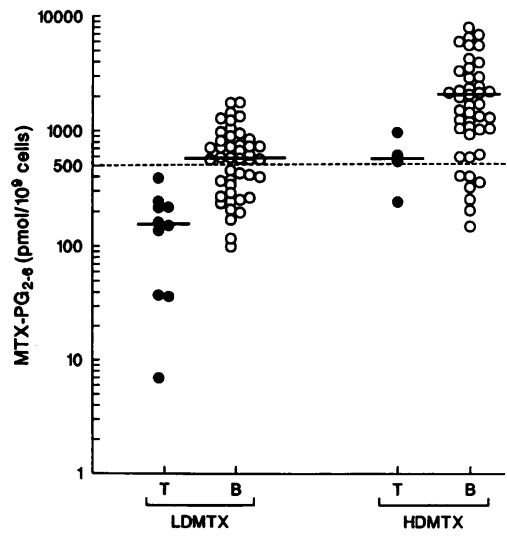

Figure 5. Intracellular MTX-PG concentrations in ALL blasts following in vivo exposure to either LDMTX or HDMTX, in children with newly diagnosed ALL. Each symbol depicts an individual patient. Open symbols depict patients with Blinage $\operatorname{ALL}(n=87)$ and closed symbols depict patients with T-lineage ALL $(n=14)$. Solid lines depict median values for each group. The dashed line represents $500 \mathrm{pmol} / 10^{9}$ blasts, the minimum intracellular MTX-PG concentration (following in vitro incubation with MTX) that was identified by Whitehead et al. (25) as associated with a more favorable outcome in children with B-lineage ALL. HDMTX achieved MTXPG concentrations above $500 \mathrm{pmol} / 10^{9}$ cells in a significantly higher proportion of patients with $\mathrm{B}$-lineage $(P=0.001)$ and $\mathrm{T}$-lineage $(P=0.01)$ ALL. $n=5$ ) versus nonhyperdiploid B-lineage blasts (median FPGS $=764 \mathrm{pmol} / \mathrm{h}$ per $\mathrm{mg}$ protein, $n=26$ ), indicating that additional mechanisms (e.g., transport or hydrolase activity) likely contribute to the ploidy differences in MTX-PG accumulation.

From a clinical perspective, it is noteworthy that HDMTX produced MTX-PG concentrations in T-lineage blasts comparable to B-lineage blasts treated with LDMTX, suggesting a strategy to at least partially circumvent the lineage differences in MTX-PG concentrations. As such, this may provide an explanation for the recently reported improved outcome of patients with T-lineage ALL treated with regimens incorporating HDMTX (e.g., $5 \mathrm{~g} / \mathrm{m}^{2}$ ) (43). The present study also establishes that higher doses of MTX are necessary in patients with nonhyperdiploid B-lineage ALL, if one wishes to achieve intracellular MTX-PG levels comparable to hyperdiploid B-lineage blasts in patients treated with low dose MTX. Collectively, these data provide a solid rationale for using HDMTX in the treatment of childhood ALL, and indicate that this may be especially important for patients with T-lineage or nonhyperdiploid B-lineage ALL, disease characteristics associated with a worse prognosis on conventional therapy.

\section{Acknowledgments}

The authors gratefully acknowledge the extensive contributions of the medical, pharmacy, and nursing staff, the excellent technical contributions of Yaqin Chu, Emily Melton, Pam McGill, Hosea Clariette, Ken Cox, Vicky Green, Gail Ubben, and Cindy Stewart, the modeling and database expertise of Yuri Yanishevski and Nancy Kornegay, the statistical assistance and expertise of Michael Hancock, Drs. Susana Raimondi and Richard Ashmun for cell ploidy determination, Dr. Fred Behm for immunophenotyping, and especially the patients and parents who participated in this study.

This work was supported in part by the following awards from the U.S. Public Health Service, National Institutes of Health, National Cancer Institute: Leukemia Program Project grant PO1-CA20180, R37CA36401, and CORE Cancer Center Support grant CA21765, and by 
a Center of Excellence grant from the State of Tennessee, an endowment from the First Tennessee Bank (W. E. Evans), and American Lebanese Syrian Associated Charities (ALSAC).

\section{References}

1. Evans, W. E., W. R. Crom, M. Abromowitch, R. Dodge, A. T. Look, P. Bowman, S. George, and C.-H. Pui. 1986. Clinical pharmacodynamics of highdose methotrexate in childhood acute lymphocytic leukemia. Identification of a relation between concentration and effect. N. Engl. J. Med. 314:471-417.

2. Niemeyer, C. M., R. D. Gelber, N. J. Tarbell, M. Donnelly, L. A. Clavell, S. R. Blattner, K. Donahue, H. J. Cohen, and S. E. Sallen. 1991. Low-dose versus high-dose methotrexate during remission induction in childhood acute lymphoblastic leukemia (Protocol 81-01 update). Blood. 78:2514-2519.

3. Camitta, B., B. Leventhal, S. Lauer, J. J. Shuster, S. Adair, J. Casper, C. Civin, M. Graham, D. Mahoney, L. Munoz, G. Kiefer, and B. Kamen. 1989. Intermediate-dose intravenous methotrexate and mercaptopurine therapy for nonT, non-B acute lymphocytic leukemia of childhood: A pediatric oncology group study. J. Clin. Oncol. 7:1539-1544.

4. Kamen, B., and A. Capdevila. 1986. Receptor-mediated folate accumulation is regulated by the cellular folate content. Proc. Natl. Acad. Sci. USA. 83:59835987.

5. Keefe, D. A., R. L. Capizzi, and S. A. Rudnick. 1982. Methotrexate cytotoxicity for L5178Y/Asn- lymphoblasts: relationship of dose and duration of exposure to tumor cell viability. Cancer Res. 42:1641-1645.

6. Kamen, B. A., and N. J. Winick. 1988. High dose methotrexate therapy: Insecure rationale? Biochem. Pharmacol. 37:2713-2715.

7. Anderson, R. G., B. A. Kamen, K. G. Rothberg, and S. W. Lacey. 1992 Potocytosis: sequestration and transport of small molecules by caveolae. Science (Wash. DC). 255:410-411.

8. Evans, W. E., M. J. Schell, and C.-H. Pui. 1990. MTX clearance is more important for intermediate-risk ALL. J. Clin. Oncol. 8:1115-1116.

9. Balinska M., Z. Nimec, and J. Galivan. 1982. Characteristics of methotrexate polyglutamate formation in cultured hepatic cells. Arch. Biochem. Biophys. 216:466-476.

10. McGuire, J. J., P. Hsieh, J. K. Coward, and J. R. Bertino. 1980. Enzymatic synthesis of folylpolyglutamates: Characterization of the reaction and its products. J. Biol. Chem. 255:5776-5788.

11. Taylor, R. T., and L. Hanna. 1977. Folate-dependent enzymes in cultured chinese hamster cells: folylpolyglutamate synthetase and its absence in mutants auxotrophic for glucine + adenosine + thymidine. Arch. Biochem. Biophys. $181: 331-344$.

12. Bertino, J. R. 1993. Karnofsky memorial lecture: ode to methotrexate. $J$. Clin. Oncol. 11:5-14.

13. Rosenblatt, D. S., W. M. Whitehead, N. Vera, A. Pottier, M. Dupont, and M.-J. Vuchich. 1978. Prolonged inhibition of DNA synthesis associated with the accumulation of methotrexate polyglutamates by cultured cells. Mol. Pharmacol. 14:1143-1147.

14. Galivan, J. 1980. Evidence for the cytotoxic activity of polyglutamate derivatives of methotrexate. Mol. Pharmacol. 17:105-110.

15. Fry, D. W., J. C. Yalowich, and I. D. Goldman. 1982. Rapid formation of polygammaglutamyl derivatives of methotrexate and their association with dihydrofolate reductase as assessed by high-pressure liquid chromatography in the Ehrlich ascites tumor cell in vitro. J. Biol. Chem. 257:1890-1896.

16. Jolivet, J., and B. A. Chabner. 1983. Intracellular pharmacokinetics of methotrexate polyglutamates in human breast cancer cells. Selective retention and less dissociable binding of 4- $\mathrm{NH}_{2}-10-\mathrm{CH}_{3}$-pteroylglutamate ${ }_{4}$ and 4- $\mathrm{NH}_{2}-10-\mathrm{CH}_{3}-$ pteroylglutamates to dihydrofolate reductase. J. Clin. Invest. 72:773-778.

17. Whitehead, V. M. 1977. Synthesis of methotrexate polyglutamates in murine L1210 leukemia cells. Cancer Res. 37:408-412.

18. Jacobs, S. A., R. H. Adamson, B. A. Chabner, C. J. Derr, and D. G. Johns. 1975. Stoichiometric inhibition of mammalian dihyrofolate reductase by the $\gamma$ glutamyl metabolite of methotrexate,4-amino-4-deoxy- $N^{10}$-methylpteroyl-glutamyl- $\gamma$-glutamate. Biochem. Biophys. Res. Commun. 63:692-698.

19. Allegra, C. J., B. A. Chabner, J. C. Drake, R. Lutz, and D. Rodbard. 1985 Enhanced inhibition of thymidylate sythetase by methotrexate polyglutamates. $J$. Biol. Chem. 260:9720-9726.

20. Allegra, C. J., J. C. Drake, J. Jolivet, and B. A. Chabner. 1985. Inhibition of phoshoribosyl-aminoimidizolecarboxamide transformylase by methotrexate and dihydrofolic acid polyglutamates. Proc. Natl. Acad. Sci. USA. 82:4881-4885.

21. Chabner, B. A., C. J. Allegra, G. A. Curt, N. J. Clendeninn, J. Baram, S. Koizumi, J. C. Drake, and J. Jolivet. 1985. Polyglutamation of methotrexate. Is methotrexate a pro-drug? J. Clin. Invest. 76:907-912.

22. Cowan, K. H., and J. Jolivet. 1984. Methotrexate-resistant human breast cancer cell line with multiple defects including diminished formation of methotrexate polyglutamates. J. Biol. Chem. 259:10793-10800.

23. Pizzorno. G., E. Mini, M. Coronello, J. J. McGuire, B. A. Moroson, A. R. Cashmore, R. N. Dreyer, J. T. Lin, T. Mazzei, P. Periti, and J. R. Bertino 1988. Impaired polyglutamation of methotrexate as a cause of resistance in CCRFCEM cells after short term, high-dose treatment with this drug. Cancer Res. 48:2149-2155.

24. McCloskey, D. D., J. J. McGuire, C. A. Russell, B. G. Rowan, J. R. Bertino, G. Pizzorno, and E. Mini. 1991. Decrease folylpolyglutamate synthetase activity as a mechanism of methotrexate resistance in CCRF-CEM human leukemia sublines. J. Biol. Chem. 266:6181-6187.

25. Whitehead, V. M., D. S. Rosenblatt, M.-J. Vuchich, J. J. Shuster, A. Witte, and D. Beaulieu. 1990. Accumulation of methotrexate polyglutamates in lymphoblasts at diagnosis of childhood acute lymphoblastic leukemia: A pilot prognostic factor analysis. Blood. 76:44-49.

26. Pui, C.-H., F. G. Behm, and W. M. Crist. 1993. Clinical and biological relevance of immunologic marker studies in childhood acute lymphoblastic leukemia. Blood. 82:343-362.

27. Pui, C.-H., W. M. Crist, and A. T. Look. 1990. Biology and clinical significance of cytogenetic abnormalities in childhood acute lymphoblastic leukemia. Blood. 76:1449-1463.

28. Look, A. T., S. L. Melvin, S. L. Williams, G. M. Brodeur, G. V. Dahl, D. K. Kalwinsky, S. B. Murphy, and A. M. Mauer. 1982. Aneuploidy and percentage of S-phase cells determined by flow cytometry correlate with cell phenotype in childhood acute leukemia. Blood. 60:959-967.

29. Williams, D. L., A. Harris, K. J. William, M. J. Brosius, and W. Lemonds. 1984. A direct bone marrow chromosome technique for acute lymphoblastic leukemia. Cancer Genet. Cytogenet. 13:239-257.

30. Mitel, F. 1991. Supplement to an international system for human cytogenetic nomenclature (ISCN). In Guidelines for Cancer Cytogenetics, F. Mitelman, editor. Karger AG, Basel, Switzerland. 1-54.

31. Rivera G. K., Raimondi S. C., Hancock, M. L., Behm F. G., Pui C.-H., Abromowitch M., Mirro J., Ochs, J., Look A. T., Murphy, S. B., Dahl G. V., Kalwinsky D. K., Evans W. E., Kun L., Simone J. V., and Crist W. M. 1991. Improved outcome in childhood acute lymphoblastic leukemia with reinforced early treatment and rotational combination chemotherapy. Lancet (N. Am. Ed.) 337:61-66.

32. D'Argenio, D. Z., and A. Schumitzky. 1979. A program package for simulation and parameter estimation in pharmacokinetic systems. Comput. Programs Biomed. 9:115-134.

33. Teresi, M. E., W. R. Crom, K. E. Choi, J. Mirro, and W. E. Evans. 1987. Methotrexate bioavailability after oral and intramuscular administration in children. J. Pediatr. 110:788-792.

34. Kamen, B. A., and N. Winick. 1986. Analysis of methotrexate polyglutamate derivatives in vivo. Methods Enzymol. 122:339-346.

35. Fabre, G., L. H. Matherly, R. Favre, J. Catalin, and J.-P. Cano. 1983. In vitro formation of polyglutamyl derivatives of methotrexate and 7-hydroxymethotrexate in human lymphoblastic leukemia cells. Cancer Res. 43:4648-4648.

36. Myers, C. E., M. E. Lippman, H. M. Eliot, and B. A. Chabner. 1975. Competitive protein binding assay for methotrexate. Proc. Natl. Acad. Sci. USA $72: 3683-3686$

37. Fleming, T. R., D. P. Harrington, and P. C. O'Brien. 1984. Designs for group sequentials tests. Controlled Clin. Trials 5:348-361.

38. Galivan, J., Z. Nimec, and M. Balinska. 1983. Regulation of methotrexate polyglutamate accumulation in vitro: effects of cellular folate content. Biochem. Pharmacol. 32:3244-3247.

39. Pui, C.-H., and W. M. Crist. 1994. Biology and treatment of acute lymphoblastic leukemia. J. Pediatr. 124:491-503.

40. Whitehead, V. M., M.-J. Vuchich, S. J. Lauer, D. Mahoney, A. J. Carroll, J. J. Shuster, D. W. Esseltine, C. Payment, A. T. Look, J. Akabutu, T. Bowen, L. D. Taylor, B. Camitta, and D. J. Pullen. 1992. Accumulation of high levels of methotrexate polyglutamates in lymphoblasts from children with hyperdiploid (>50 chromosomes) B-lineage acute lymphoblastic leukemia: A Pediatric Oncology Group study. Blood. 80:1316-1323.

41. Goker, E. J. T. Lin, T. Trippett, Y. Elisseyeff, W. P. Tong, D. Niedzwiecki, C. Tan, P. Steinherz, B. I. Schweitzer, and J. R. Bertino. 1993. Decreased polyglutamylation of methotrexate in acute lymphoblastic leukemia blasts in adults compared to children with this disease. Leukemia (Baltimore). 7:1000-1004.

42. Barredo J. C., T. W. Synold, J. Laver, M. V. Relling, C.-H. Pui, D. G. Priest and W. E. Evans 1994. Differences in constitutive and post-methotrexate folylpolyglutamate synthetase activity in B-lineage and T-lineage leukemia. Blood. 84:564-569.

43. Feickert, H. J., C. Bettoni, M. Schrappe, A. Reiter, W.-D. Ludwig, U. Bode, W. Ebell, and H. Riehm. 1993. Event-free survival of children with T-cell acute lymphoblastic leukemia after introduction of high dose methotrexate in multicenter trial ALL-BFM 86. Proc. Am. Soc. Clin. Oncol. Annu. Meet. 12:317 (Abstr). 